\title{
Mechanism of ultrasound strengthening preparation nanomaterials
}

\author{
Yong-guang Bi, Xu-si Xu, Shi-ting Deng, Hong Yu, Xue-wei Chen, Xue-mei \\ Liu , Hai-lan Huang
}

School of Pharmacy, Guangdong Pharmaceutical University, Guangzhou 510006, Guangdong, China

E-mail:biyongguang2002@163.com

Keywords: ultrasound; hydroxyapatite; preparation; mechanism

\begin{abstract}
This paper will discuss strengthening mechanism of ultrasonic hydroxyapatite nm from each ultrasound parameters, such as: research ultrasonic power, intensity, frequency, etc., the findings concluded that ultrasound is through a liquid medium to a solution of molecules, ions action. When the ultrasound propagation in a liquid medium and the energy reaches a certain threshold, it will have a "cavitation" phenomenon. Thus, the formation of cavitation nuclei and growth has played a significant role in promoting, ultrafine, uniform nano-powders obtained provides a good foundation, it has been widely used in the preparation of nanometer powder.
\end{abstract}

\section{Introduction}

Nanomaterials refers to the space between the single crystal size between $1 \mathrm{~nm}-100 \mathrm{~nm}$ range or a single crystal by material effect constitute a certain space, and its scope is very broad, including ultra various metals, non-metallic, inorganic compounds and organic compounds fine powder. Nano materials are available from the dimension, composition relative to the number of different angles, etc. and conductive properties, usually in dimension classified into zero-dimensional, one-dimensional and two-dimensional. The system has a zero-dimensional nanoparticles, clusters, etc $^{[1]}$; a one-dimensional system has nanowires (Nanosilk), nanorods (Nanorods), nanotubes (Nanotubes); two-dimensional nano material is mainly nanofilms (Nano-Membrane); Because these units often have quantum characteristics, so the zero-dimensional, one-dimensional and two-dimensional nanomaterials and basic structural units are called quantum dots (quantum dot), quantum wire (quantum wire) and the quantum well (quantum well). Because quantum affinity between the structural units or synergistic enhancement of the role of nanomaterials in general unparalleled material properties, has broad applications in photoelectrochemical properties, etc., aroused great interest of researchers at home and abroad and widespread concern . Currently, nano-science has become colloid chemistry, condensed matter physics, atomic physics, Solid State Chemistry, chemical reaction kinetics and surface, interface, and other interdisciplinary scientific gathering and the emergence of a comprehensive discipline. The last century, scientists praise: nanotechnology research in the 21 st century will become the most development potential.

Ultrasound chemical, also known as sonochemistry, is cross-penetration and developed mutual chemistry between physical acoustics and a new discipline, as early as 1895, scientists observed Bamaby and Thormycroft on sunken submarine propeller erosion, published in human history the first report on cavitation, 1927, Loomis was first reported ultrasound has the effect of speeding up the rate of biochemical reactions, but until the 1980s Sonochemical science really began to board the stage of history, the beginning of the formation of a new chemical branch, for two reasons: First, in terms of the source of ultrasound, because the development of science and technology, all kinds of efficient and economical ultrasonic source to be produced; the second is the demand side of the new functional materials, people have the characteristics of functional materials demand more and more, the process for the preparation of materials have become increasingly demanding ${ }^{[2-4]}$. Currently, the main research and application of ultrasound technology involves chemistry, photoelectric chemistry, inorganic chemistry, nano-materials and the development of new materials have been widely used in the synthesis, wastewater treatment, preparation of ceramic powders and 
materials science.

In practical applications, we use ultrasound on the reaction solution was subjected to irradiation, fu as can be divided into two categories: direct and indirect ultrasound ultrasound. Indirect ultrasonic bath type ultrasonic reactor, mainly used for cleaning. The principle is - exists in the liquid under the action of microbubbles vibration sound waves, when the sound pressure reaches a certain value, the rapid growth of the bubble, then suddenly closed, resulting in a shock wave in the bubble is closed, to achieve the goal of cleaning ${ }^{[5]}$. Direct ultrasound probe system type reactor is, such a device is an ultrasonic probe directly immersed in the reaction liquid, the energy directly into the reaction system, compared to the indirect ultrasound, ultrasound directly reach a larger reaction vessel ultrasonic power, it can be a lot of ultrasonic energy in a reaction solvent, and can more easily modify the experimental parameters, but because of the ultrasonic probe in direct contact with the reaction solution, it is possible to introduce impurities and contamination, so before each experiment, the application of ethanol cleaning probe, then it drying.

Hydroxyapatite (HAP) is prevalent in the Earth's surface, in human and animal bones and teeth were found in its present form salts, so the organism has good compatibility and have biological activity, is a good bone tissue replacement materials, and is widely used in biotechnology, for example, be used as an alternative to bone, the bone void filler, bone cement, coating implants, has now become the world clinical orthopedic surgeon like to use materials with potential prospects ${ }^{[6-10]}$. Research shows that hydroxyapatite can remove specific chemical pollution, improve food hygiene and water purification to remove contaminants such as metal ions in water and can absorb some of the organic substances that can improve the efficiency of the drug, adsorption characteristics of hydroxyapatite It has an important application value and theoretical significance.

Although nano-hydroxyapatite good biocompatibility, inorganic chemical composition close to the bone of the chemical composition, but its drawback is that poor mechanical properties, brittle, compressive strength and flexural strength is not enough. And the lack of natural human collagen tissue components, etc., it is necessary to use an organic - inorganic composite material prepared way to obtain high-strength, biocompatible scaffold for bone tissue better meet biomedical needs $^{[11-14]}$. Secondly, nano-hydroxyapatite composite as slow release drug delivery and treatment of industrial waste water as adsorbents and other aspects also play an important role.

In summary, this paper will discuss strengthening mechanism of ultrasonic hydroxyapatite nm from each ultrasound parameters, such as: research ultrasonic power, intensity, frequency, etc., the findings concluded that ultrasound is through the liquid medium of the solution molecules, ions have an effect. When the ultrasound propagation in a liquid medium and the energy reaches a certain threshold, it will have a "cavitation" phenomenon.

\section{Materials, reagents and methods}

\section{Instrument}

752 UV-Vis spectrophotometer, AY120 electronic analytical balance, AY120 electronic analytical balance, KH-400KDB CNC high-power ultrasonic cleaner, digital temperature drying oven, D8-ADVANCE X-ray powder diffraction, TENSOR-37 fourier Fourier transform infrared spectrometer.

\section{Reagents}

$\mathrm{Ca}\left(\mathrm{NO}_{3}\right)_{2} \bullet 4 \mathrm{H}_{2} \mathrm{O},(\mathrm{NH} 4)_{2} \mathrm{HPO}_{4}$, ammonia, acetaminophen, phenol and ethanol.

\section{The pre-reaction HAP sample preparation}

Control experiments $\mathrm{Ca}\left(\mathrm{NO}_{3}\right)_{2} \cdot 4 \mathrm{H}_{2} \mathrm{O}$ and the molar ratio of $(\mathrm{NH} 4)_{2} \mathrm{HPO}_{4}$ is 1.67. Specific experimental procedure is as follows: 5: 3 molar ratio of calcium nitrate solution were prepared and diammonium phosphate solution: Weigh $11.808 \mathrm{gCa}\left(\mathrm{NO}_{3}\right) 2 \cdot 4 \mathrm{H}_{2} \mathrm{O}$, and $3.962 \mathrm{~g}(\mathrm{NH} 4)_{2} \mathrm{HPO}_{4}$, were dissolved in deionized water, formulated as $0.050 \mathrm{~mol} / \mathrm{L}$ of $\mathrm{Ca}\left(\mathrm{NO}_{3}\right)_{2}$ solution and $0.030 \mathrm{~mol} / \mathrm{L}$ of (NH4) ${ }_{2} \mathrm{HPO}_{4}$ solution. 


\section{Preparation of nano-HAP under ultrasound}

Amount of the same volume of the above with a good solution $20 \mathrm{~mL}$ of two, both with aqueous ammonia to adjust the $\mathrm{pH}$ of the solution was 10 to 10.5 , and the auxiliary ultrasonic vigorous mechanical stirring (ultrasonic power: $\mathrm{W}=400 \mathrm{~W}$, mechanical agitation speed: $\mathrm{n}=200$ switch/min) of the latter $\left(0.030 \mathrm{~mol} / \mathrm{L}\right.$ of $(\mathrm{NH} 4){ }_{2} \mathrm{HPO}_{4}$ solution $)$ was slowly added dropwise $(<10 \mathrm{~mL} / \mathrm{min})$ using a separatory funnel and constantly stirred calcium nitrate solution, and treated with aqueous ammonia to control the $\mathrm{pH}$ value of 10 to 10.5 , the reaction temperature is room temperature $25^{\circ} \mathrm{C}$. Until the addition was complete, continue to ultrasound and to assist with vigorous mechanical stirring $2 \mathrm{~h}$, and then the reaction product was allowed to stand for aging for about $12 \mathrm{~h}$, the supernatant decanted lower sediment centrifugation, thoroughly washed with deionized water centrifugal separator 3 to 5 times, each time $15 \mathrm{~min}$, to remove the ammonium ion, until neutral, after which the product was oven dried transverse temperature $80^{\circ} \mathrm{C} 12 \mathrm{~h}$ to give HAP powder sample denoted as S2.

\section{Results and Discussion}

\section{Mechanism of ultrasound}

Maniatis $\mathrm{C}$ believes that without a critical synthesis of powder agglomeration is to control the crystal growth process of reunion. Commonly used anti-agglomeration methods are: washing with an organic solvent, azeotropic distillation dehydration, ultrasound, use surfactants, which bounce effect is an effective method to reduce particle agglomeration, its mechanism of action thought to cavitation effects.

By acoustic cavitation improve reaction yields and lead to a new discipline of chemical reactions, reflect a very unique interaction between energy and matter. It refers to a liquid acoustic cavitation nuclei formed tiny bubbles, vibration, grow, shrink to collapse, and the physical and chemical changes induced. When cavitation bubbles collapse in a very short time a very small space around the cavitation bubble, the instant high temperature $(5000 \mathrm{~K})$, high pressure $(1.8 \times 108 \mathrm{~Pa})$ and more than $1010 \mathrm{~K} / \mathrm{s}$ cooling rate, accompanied by a strong shock wave and speed of $400 \mathrm{~km} / \mathrm{h}$ and discharge light jet effect. These conditions are sufficient to break the chemical bonds in the compound occurs cavitation nuclei, the aqueous phase of combustion or thermal decomposition, and can promote heterogeneous interfacial disturbance between the phase interface update and generate new phase, thus accelerating the mass transfer and the interface between the transmission thermal process.

In addition, the ultrasonic cavitation increases the chemical reactivity, increase medium particle collision probability between. High temperatures and lots of tiny bubbles in the surface of solid particles generated ultrasonic cavitation generated also greatly reduces the specific surface free energy of small grains, inhibit aggregation nuclei and grow up. Ultrasonic cavitation generated shock waves and microjets shear crushing action of the particles on the effective destruction of the reunion between the nuclei or particles, controlling the size of the particles. Meanwhile, the ultrasonic irradiation significantly increased the nucleation density, promote the formation and growth of nuclei. In the nucleation stage, the critical nucleus formation requires a certain Serve nuclear energy, heat generated by the ultrasonic cavitation nuclei make the system easier to achieve the desired stability of Guinea into nuclear energy, nucleation probability increases, the nucleation rate increases and instantly generate a large number of ultra-fine nuclei in the system. Nucleation stage of growth to the collapse of cavitation bubbles can cause a reaction liquid microscopic vortex, cavitation bubbles per second, hundreds of millions on such a whirl on the very significant cause agitation in the reaction solution..Thus, the formation of cavitation nuclei and growth has played a significant role in promoting, ultrafine, uniform nano-powders obtained provides a good foundation, it has been widely used in the preparation of nanometer powder. 


\section{Conclusions}

The results concluded that ultrasound is through a liquid medium to a solution of molecules, ions have an effect. When the ultrasound propagation in a liquid medium and the energy reaches a certain threshold, it will have a "cavitation" phenomenon. Thus, the formation of cavitation nuclei and growth has played a significant role in promoting, ultrafine, uniform nano-powders obtained provides a good foundation, it has been widely used in the preparation of nanometer powder.

\section{Acknowledgements}

This work was financially supported by Guangdong Department of Water Resources Science and Technology Innovation Project (No. 2015-20).

\section{References}

[1]R.Scllnlltt,A.B.Miller,C.Chemla,PhysRevB,1978,35(15):8113.

[2]Y.Lu,P.K.Liaw,JoumalofPetroleumScienceandTechnology,,2001, 53(3):31.

[3]L.E.Brus,J.Chem.Phys.,1991,95:523.

[3]Ghasemi,M.F.Mousavi,etal.,Ultrasonies Sonoehemistry,2008,15:448.

[4]H.B.Jin,K.5.Susliek,J.Am.Chem.Soe.,2007,129(8),:2242.

[5]ABD EL-GAWAD. Repair of fragmented DNA and treatment of heavy metal intoxication by intrarenous injection of nano-Hydroxyapatite: WO, 2011/124234 [P] . 2011-10-13.

[6]University of ARKANSAS. Use of advanced nanomaterials for increasing sepecific cell functions: US, $2010 / 104652$ [P] . 2010-04-29

[7]Clemson University Research Foundation. Method and compositions for temporal release of agents from a biodegradable scaffold:US,2011/038921 [P] . 2011-01-17.

[8] Rose FR, Oreffo RO. Bone tissue engineering: hope vs hype.Biochem Biophys Res Commun. 2002;292(1):1-7.

[9] LeGeros RZ. Properties of osteoconductive biomaterials:calcium phosphates. Clin Orthop Relat Res. 2002;(395):81-98.

[10] Nishida J, Shimamura T. Methods of reconstruction for bonedefect after tumor excision: a review of alternatives. Med SciMonit. 2008;14(8):RA107-113.

[11] Nandi SK,Kundu B,Ghosh SK,et al.Efficacy of nano-hydroxyapatite prepared by an aqueous solution combustion technique in healing bone defects of goat.J Vet Sci. 2008; 9(2): 183-191.

[12] Petite H, Viateau V, Bensaïd W, et al. Tissue-engineered bone regeneration. Nat Biotechnol. 2000;18(9):959-963.

[13]Xu Q,Lu HY, Zhang JC, etal. Tissue engineering scaffold material of porous nanohydroxyapatite/polyamide 66. Int J Nanomedicine. 2010; 5: 331-335.

[14] Shi Z, Huang X, Cai Y,et al. Size effect of hydroxyapatite nanoparticles on proliferation and apoptosis of osteoblast-like cells. Acta Biomater. 2009;5(1):338-345. 